\title{
Origin of power-law X-ray emission in the Steep power-law state of X-ray Binaries
}

\author{
Jiancheng Wang and Lihong Yan ${ }^{1}$ \\ Yunnan Observatory /National Astronomical Observatories, Chinese Academy of Sciences, \\ P.O. Box 110 Kunming, Yunnan Province 650011, P.R. China
}

Received —

${ }^{1}$ Graduate School, Chinese Academy of Sciences, Beijing, P.R. China 


\begin{abstract}
We present a new scenario of the emissive origin in the Steep Power Law (SPL) state of X-ray Binaries. The power-law component of X-ray emission is the synchrotron radiation of relativistic electrons in highly magnetized compact spots orbiting near the inner stable circular orbit (ISCO) of black hole and has a hard spectrum that extends to above $\mathrm{MeV}$ bands determined by electron acceleration rate. These photons are then down-scattered by the surrounding plasma and form an observed steep spectrum. The relevance of this model with high-frequency quasi-periodic oscillations (HFQPOs) and extremely high luminosity of the SPL state is discussed.
\end{abstract}

Subject headings: accretion, accretion disks-black hole physics-radiation mechanisms: nonthermal-X-rays: binaries 


\section{Introduction}

The physical origin of the SPL state generating HFQPOs, extremely high luminosity and spectra that extend to above $\mathrm{MeV}$, remains one of the outstanding problems in Black-hole binaries (BHBs). Most models on the spectra of the SPL state invoke inverse Compton scattering of seed photons from the disk in a nonthermal corona (Zdziarski 2004; Zdziarski et al. 2005). The origin of the nonthermal electrons has

led to the models with complicated geometry and feedback mechanisms. Alternative models involve bulk motion Comptonization in the context of a converging subKeplerian flow within $50 R_{g}$ of the Black Hole (BH) (Titarchuk \& Shrader 2002; Turolla et al. 2002). The frequencies of HFQPOs are as fast as the dynamical ones at the ISCO of Schwarzschild BHs. The models of HFQPOs involve the resonance oscillation modes occurring in specific radii where the radiating source orbit has the coordinate frequencies scaling with a defined ratio in the Kerr metric (Merloni et al. 1999; Abramowicz \& Kluźniak 2001; Remillard et al. 2002; Abramowicz et al. 2003; Kluźniak et al. 2004; Schnittman \& Bertschinger $\quad$ 2004; Török et al. 2004; Schnittman 2005; Schnittman \& Rezzolla 2006). In Schnittman \& Bertschinger (2004) and Schnittman (2005) they have developed a geodesic hot spot model to explain the X-ray light curves, in which a collection of "hot spots", small region of excess emission move on geodesic orbits near the ISCO. The hot spot model is characterized by the black hole mass and spin, the disk inclination angle, and the simple properties of the hot spot (such as size, shape and overbrightness).

Current models of the SPL state are deficient in terms of specifying the radiation mechanisms that would imprint a given oscillation mode into the X-ray light curve, such as the radiation component of hot spots. In fact the HFQPOs are commonly tied to a hard X-ray component, rather than the thermal component that can be directly attributed to the 
accretion disk. It is unlikely that the HFQPOs are coming from a cool, thermal spot getting upscattered by a hot corona. From the photon spectra of the SPL state, there appears to be a hot corona with Compton parameter $y \sim 1$, the lower energy bands $(2-6 \mathrm{KeV})$ have the smaller amplitude modulation. It is difficult to explain the larger significance of HFQPO detections in the higher energy bands $(6-30 \mathrm{KeV})$ relative to the signal in the lower energy bands $(2-6 \mathrm{KeV})$.

In this paper, we propose a new scenario that the X-rays are the synchrotron radiation of highly magnetized compact spots orbiting near the ISCO, and have a hard spectrum that extends to the $\mathrm{MeV}$ bands determined by electron acceleration rate. These photons are then down-scattered by the surrounding plasma and form an observed steep spectrum. In the innermost disc region the spot moves like a test particle in the black hole field and modulates the observed X-ray flux with HFQPOs through its synchrotron emission. The coordinate frequencies of the orbital motion with a defined ratio present a pair of HFQPOs (Schnittman \& Bertschinger 2004; Schnittman 2005). In section 2 we present the basic relations determining the synchrotron radiation of a highly magnetized compact spot orbiting near the ISCO and Compton scattering mechanism of the SPL formation. In section 3 we conclude a discussion of open question on the SPL state based on new scenario.

\section{Synchrotron Emission and Compton Scattering Mechanisms}

We assume that a radiation spot with the size $R_{b}$ in the comoving frame, has a Doppler factor $\delta$ due to its motion relative to the observer. In the observer frame, the total energy of the spot is related to the comoving energy density $\varepsilon$ by $E \simeq \varepsilon R_{b}^{3} \delta$. If a fraction $f$ of this energy is radiated during the observed timescale $R_{b} /(c \delta)$, the observed luminosity is given by

$$
L \simeq 4 \varepsilon f R_{b}^{2} c \delta^{4}
$$


where the factor of $\delta^{2}$ comes from the observed power with beaming effect. We assume that a fraction $\varepsilon_{B}$ of the energy density $\varepsilon$ is magnetic, we then obtain the magnetic field

$$
B \simeq\left(\frac{8 \pi \varepsilon_{B} L}{f R_{b}^{2} c \delta^{4}}\right)^{1 / 2}
$$

and the ratio of the radiative cooling time $t_{s y n}$ to the dynamical timescale $t_{d}$,

$$
\frac{t_{\text {syn }}}{t_{d}}=\frac{6 \pi m_{e} c}{\sigma_{T} \gamma B^{2}}=3.0 \times 10^{-3}\left(\frac{L}{L_{E d d}}\right)^{-1}\left(\frac{R_{b}}{R_{g}}\right)\left(\frac{f}{\varepsilon_{B}}\right) \gamma^{-1} \delta^{4},
$$

where $L_{E d d}$ is the Eddington luminosity of the black hole, $R_{g}$ is the Schwarzschild radius and $\gamma$ is the Lorentz factor of the electron. The condition of efficient synchrotron is that the dynamical time of electrons $t_{d}$ is larger that the synchrotron cooling time of electrons $t_{d y n}$. If $\varepsilon_{B}$ and $f$ are the same amount and $L / L_{E d d}$ is not small, the synchrotron radiation of relativistic electrons will become an effective mechanism of X-ray radiation in a highly magnetized compact spot with the size $\Delta R \sim R_{g}$ near the ISCO. We can assess whether the Doppler factor $\delta$ is large through the velocity $v_{\phi}$ of the spot orbiting near the ISCO,

$$
v_{\phi}=\frac{r \sin i}{2 \pi\left(r^{3 / 2} \pm a\right)},
$$

where $a$ is the spin momentum of black hole, $i$ is the inclination angle of the spot orbiting plane with respect to the spin axis of black hole. We then obtain that the maximum velocity observed is less than ten percent of light speed. Therefore the beaming effect is not important. In the following discussion, we ignore the beaming effect. The electrons of the spot orbiting near the ISCO are assumed to be accelerated by a specific mechanism. The spectrum of the accelerated electrons determines the energy spectrum of synchrotron radiation. The most general assumption for the spectrum of accelerated electrons is the power-law spectrum with an exponential cutoff at energy $\gamma_{0}$ :

$$
N(\gamma)=N_{0} \gamma^{-\alpha} \exp \left(-\gamma / \gamma_{0}\right)
$$

The energy cutoff $\gamma_{0}$ in the spectrum of electrons is determined by the balance between the electron acceleration and cooling times. The acceleration time of electrons is usually given 
by the following general form:

$$
t_{a c c}=\eta \frac{\gamma m_{e} c}{e B_{\perp}}
$$

Hereafter it is assumed that the magnetic field is distributed isotropically, e.g. $B_{\perp}=\sqrt{2 / 3} B$. The parameter $\eta \geq 1$ presents the rate of acceleration which could be the energy-dependent. In different astrophysical environments, $\eta$ remains a rather uncertain model parameter due to highly unknown acceleration mechanism. The synchrotron cooling time is given by

$$
t_{s y n}=\frac{6 \pi m_{e} c}{\sigma_{T} \gamma B^{2}}
$$

Then the energy cutoff $\gamma_{0}$ is determined by the condition $t_{a c c}=t_{s y n}$ :

$$
\gamma_{0}=\sqrt{\frac{6 \pi e}{\sigma_{T}}} B^{-1 / 2} \eta^{-1 / 2}
$$

The high energy cutoff of synchrotron emission is given by

$$
\epsilon_{0}=\left(\frac{3}{2}\right) \frac{e B h \gamma_{0}^{2}}{2 \pi m_{e} c}=\frac{9}{4 \alpha_{f}} m_{e} c^{2} \eta^{-1}=160 \eta^{-1} M e V,
$$

which depends only on the parameter $\eta$, where $\alpha_{f}=1 / 137$ is fine-structure constant. Thus, in the regime of acceleration with $\eta \leq 100$, the synchrotron radiation emitted by a highly magnetized compact spot can result in an effective production of hard X-rays that extend to above MeV bands. The non-relativistic or relativistic shock acceleration is most widely accepted theory for the acceleration of nonthermal particles (Blandford \& Eichelar 1987; Heavens \& Drury 1988; Kirk et al. 2000). The produced electron spectrum has the spectral index of $\alpha \simeq 2.5-3.0$, typically found in the optically thin synchrotron emission of AGNs. The hard X-rays created by electron synchrotron emission has the photon index of $\alpha_{p} \simeq 1.7-2.0$, which is not consistent with the observed index of SPL state.

In fact the hard X-ray synchrotron photons will be down-scattered by the surrounding corona and form a softer spectrum where the photon index increases by unit $\alpha_{p} \rightarrow \alpha_{p}+1$. Since the energy of hard photons is higher than the electron energy in the corona, the 
Comptonization is described by the following equation (Sunvaev \& Titarchuk 1980)

$$
\frac{1}{Z^{2}} \frac{d}{d Z} Z^{4} N-\beta N=-\beta f(Z) / Z^{3},
$$

where $Z=\frac{h \nu}{m_{e} c^{2}}, \beta^{-1}=\frac{3}{\pi^{2}}\left(\tau_{0}+\frac{2}{3}\right)^{2}$ corresponding to the mean number of scatterings, $\tau_{0}$ is the optical depth through the corona, and $f(Z)$ is the original spectrum of the photons. The solution of the above equation is as follows:

$$
F_{\nu}(Z)=\frac{\beta}{Z} \exp (-\beta / Z) \int_{Z}^{\infty} f(Z) \exp (\beta / \zeta) \frac{d \zeta}{\zeta} .
$$

For a power law spectrum of the hard photons $f(Z) \propto Z^{-\alpha_{p}}$ with $Z / \beta \gg 1$ corresponding to large optical depth $\left(\tau_{0} \gg 1\right)$ and high energy photon $(Z \sim 1)$, we have the Compton scattered spectrum $F_{\nu}(Z) \propto Z^{-\alpha-1}$ that can explain the observed spectra with the photon index of $2.4-3.0$.

For hard X-ray synchrotron photons $(h \nu \gg 1)$, the net effect of Compton scattering process is a transfer of energy from the photon to the electron. The average fractional energy change per scattering is large for high energy photons, the spectral shape at higher energy bands is seriously affected by Compton scattering. It implies that most HFQPOs appear more significantly in higher energy bands. The light curves actually have large amplitude fluctuations in the higher energy bands, as these scattered photons get smoothed out less in time. Furthermore the higher harmonic modes are successfully damped in the scattering process, so is the fundamental peak (Schnittman \& Rezzolla 2006).

\section{DISCUSSION}

We have proposed a new scenario of the emissive origin in the SPL state. Our model is based on two standard processes, in which the X-ray photons with a power law spectrum extending to above $\mathrm{MeV}$ bands is firstly produced by the synchrotron 
radiation of highly magnetized compact spots orbiting near the ISCO and then these photons are down-scattered by the thermal electrons of surrounding corona to form an observed steep spectrum. The high energy cutoff of the spectrum is only determined by the electron acceleration rate in the spot. As in the model, we show that the spot must be small $\left(R_{b} \sim R_{g}\right)$ and high magnetic to become an efficient synchrotron source. Since the efficiency and the absolute flux $\left(\propto R_{b}^{2} B^{3}\right)$ of synchrotron radiation contributing to X-ray power law component depend on the luminosity $L / L_{E d d}$. It implies that the SPL state tends to dominate BHB spectra as the luminosity approaches the Eddington limit (McClintock \& Remillard 2006).

In the compact spot, the synchrotron emission and Compton scattering mechanisms appear capable of producing the SPL spectrum. As the hot spot model proposed by Schnittman \& Bertschinger (2004) and Schnittman (2005), the synchrotron spots with different lifetimes move on geodesic orbits near the ISCO, they can produce the X-ray light curve with the HFQPO power spectra. It is why should the HFQPOs appear in the SPL state and not others. The high energy photons tend to transfer energy into the electrons in the surrounding plasma to form steeper energy spectra, measuring the energy spectra of the different QPOs peaks would prove extremely valuable in understanding the emission and scattering mechanisms. There are some important messages in the relationship between the power-law emission and the QPO properties. In the sources that exhibit the HFQPOs with a 3:2 frequency ratio, the low frequency QPO appears when the power law flux is very strong, whereas the high frequency appears when the power law flux is weaker (Remillard et al. 2002, 2006). This implies that the power law photons most likely are coming emissive spots, and are affected by Compton scattering as these scattered photons from the spots of outward orbits with low frequency QPO get smoothed out less. The fact that the HFQPOs are seen most clearly in high energy bands challenges that the hard X-ray photons are coming from the thermal photons of accretion disk getting upscattered 
by a hot corona. Since the Compton scatterings seriously damp the amplitude modulation of the light curves in high energy bands.

We acknowledge the financial supports from the National Natural Science Foundation of China 10673028 and 10778702, and the National Basic Research Program of China (973 Program 2009CB824800). 


\section{REFERENCES}

Abramowicz, M.A., Kluźniak, W. 2001, A\&A, 374, L19

Abramowicz, M.A. et al. 2003, PASJ, 55, 467

Blandford, R., Eichelar, D. 1987, Phys. Rep., 154, 1

Heavens, A.F., Drury, L. O’C., 1988, MNRAS, 235, 997

Kirk, J.G., Guthmann, A.W., Gallant, Y.A., Achterberg, A. 2000, ApJ, 542, 235

Kluźniak, W., Abramowicz, M.A., Lee, W. 2004, AIPC, 714, 379

McClintock, J.E., Remillard, R.A. 2006, in Compact Stellar X-ray Sources, ed. W.H.G.

Lewin, M. van der Klis (Cambridge: Cambridge Univ. Press), 157 (astro-ph/0306213)

Merloni, A., Vietri, M., Stella, L., Bini, D. 1999, MNRAS, 304, 155

Remillard, R.A., Muno, M.P., McClintock, J.E., Orosz, J.A. 2002, ApJ, 580, 1030

Remillard, R.A., McClintock, J.E., Orosz, J.A., Levine, A.M. 2006, ApJ, 637, 1002

Schnittman, J.D., Bertschinger, E. 2004, ApJ, 606, 1098

Schnittman, J.D. 2005, ApJ, 621, 940

Schnittman, J.D., Rezzolla, L. 2006, ApJ, 637, 1113

Sunyaev, R.A., Titarchuk, L.G., A\&A, 86, 121

Titarchuk, L., Shrader, C. 2002, ApJ, 567, 1057

Török, G., Abramowicz, M.A., Kluźniak, W., Stuchlík, Z. 2004, A\&A, 436, 1

Turolla, R., Zane, S., Titarchuk, L. 2002, ApJ, 576, 349 
Zdziarski, A.A., Gierlinński, M. 2004, PThPS, 155, 99

Zdziarski, A.A. et al. 2005, MNRAS, 360, 825 